\title{
Analytical Sciences
}

\author{
Peter C. Hauser*
}

\begin{abstract}
The research group for analytical and bioanalytical sciences at the Department of Chemistry of the University of Basel is working on miniaturization of analytical techniques, with special focus on detection. Downsizing is a challenge for quantitation and contributions have been made based on different techniques which encompass atomic and molecular spectroscopy as well as different electroanalytical methods. Particularly successful has been contactless conductivity detection and an introduction to this technique is given as an example of the activities of the research group.
\end{abstract}

Keywords: Analytical sciences · Capillary electrophoresis · Contactless conductivity detection $\left(C^{4} D\right)$. Lab-on-chip · Miniaturization

\section{Contactless Conductivity Detection for Microseparation Techniques}

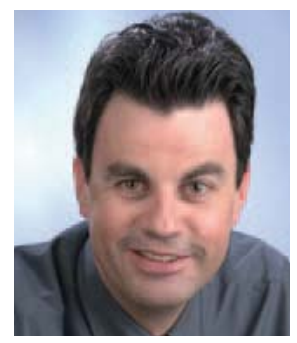

Recent scientific achievements in genomics, proteomics and other branches of the life sciences are largely due to advances in the analytical sciences brought about by down-scaling of separation methods. In the last consequence this leads to socalled lab-on-a-chip devices, but more conventional approaches, such as HPLC and capillary electrophoresis have also strongly profited from this trend to miniaturization. However, down-scaling imparts an on-going challenge to quantification, due to the significant reduction of the cell volume. To meet this detection challenge, the research group of Peter Hauser has pursued different approaches such as plasma atomic emission spectroscopy in capillaries, ${ }^{[1-4]}$ amperometric detection in electrophoresis carried out in conventional capillaries ${ }^{[5]}$ as well as on lab-on-chip devices, ${ }^{[6,7]}$ and using novel light-emitting diodes for the deep-UV range. ${ }^{[8,9]}$ In recent years, the main focus has been on capacitively coupled contactless conductivity detection $\left(C^{4} D\right)$. This method is very versatile and applications in areas as diverse as clinical analysis, the life sciences, separation of enantiomers, environmental field analysis, process monitoring etc. have been investigated.

In $\mathrm{C}^{4} \mathrm{D}$ devices the measurement is carried out through the walls of a measuring cell using external applied electrodes. This is possible as an electrical double layer is established with this arrangement. The electrical charge contained in the electrode together with the assembled ions of opposite charge in the solution form a capacitance. The situation is illustrated in Fig. 1. In Fig. 1A the well known electrical double layer obtained at a normal electrode in contact with the solution is shown. For isolated electrodes, as shown in Fig. 1B, the two layers are separated by the dielectric and thus the resulting capacitances are much smaller. A typical cell geometry based on a pair of tubular electrodes arranged axially and

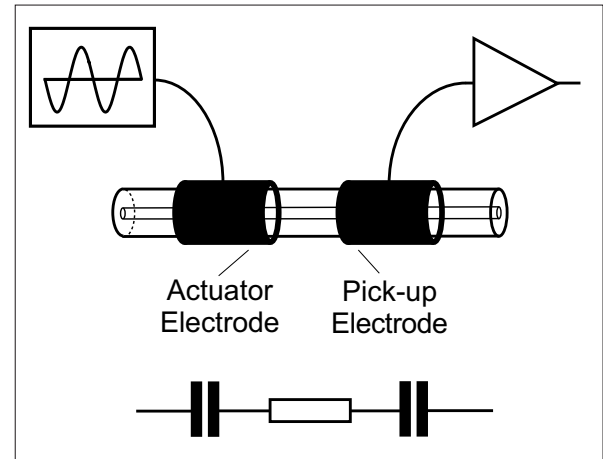

Fig. 2. Tubular electrodes on a capillary filled with an electrolyte and the resulting simplified equivalent circuitry.

encompassing a capillary is shown in Fig. 2 along with the (simplified) equivalent circuitry consisting of a serial combination of capacitor-resistor-capacitor.

The conductance of the solution (due to the movement of ions) can be probed by applying a voltage and measuring the resulting current, but ac-voltages have to be employed as the capacitances block dc-voltages. The impedance of a $C^{4} \mathrm{D}$ cell can be approximately described with
A
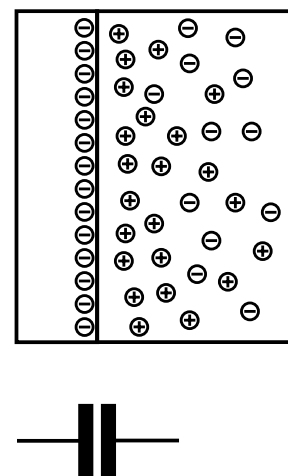

B

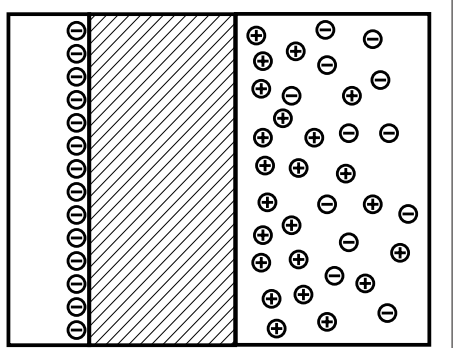

${ }^{\star}$ Correspondence: Prof. P. C. Hauser

Department of Chemistry

University of Basel

Spitalstrasse 51

$\mathrm{CH}-4056$ Basel

E-mail: peter.hauser@unibas.ch
Fig. 1. Electrolytic double layers formed through the attraction of cations from a solution at negatively polarized electrodes leading to the creation of capacitances. A) The usual case for electrodes in direct contact with the electrolyte. B) Electrodes isolated from the solution with a dielectric. 
Eqn. (1) where $i$ is the cell current, $V$ the applied voltage, $R$ the solution resistance, $C$ the double layer capacitances and $f$ the applied frequency.

$$
i=\frac{V}{\sqrt{R^{2}+\left(\frac{1}{2 \pi f C}\right)^{2}}}
$$

An examination of Eqn. (1) shows that if the product of frequency and capacitance is large, then the associated term becomes negligible and the equation simplifies to the usual form of Ohm's law. Thus in order to determine the resistance (the inverse of the conductance) high frequencies have to be employed. For the miniature cells used, the cell capacitances are exceedingly small (typically $100 \mathrm{fF}$ ) and thus relatively high frequencies $>300$ $\mathrm{kHz}$ are needed. For modern electronics this is not a hindrance, however. Fundamental studies carried out in the Hauser group $^{[10-12]}$ led to commercially available $\mathrm{C}^{4} \mathrm{D}$-cells for capillaries and lab-on-chip devices (www.edaq.com). [13]

$\mathrm{C}^{4} \mathrm{D}$ was developed for capillary electrophoresis (CE) as an alternative detection method. Commercial instruments are fitted with UV-absorbance detectors but this is not well suited for all analytes since nonabsorbing organic species, as well as most inorganic ions can only be determined with indirect methods. The internal diameters of the capillaries for optical detection are usually 100 or $75 \mu \mathrm{m}$, smaller capillaries are not feasible as not only the pathlengths become too small, but also the cross-section of the apertures have to become smaller, significantly reducing the transmitted light intensity. With $C^{4} \mathrm{D}$ a further reduction is, however, not problematic. As is illustrated in Fig. 3, in a study of the effect of the internal diameter of the capillary on the separation efficiency it was found that, as expected, a strong improvement can be

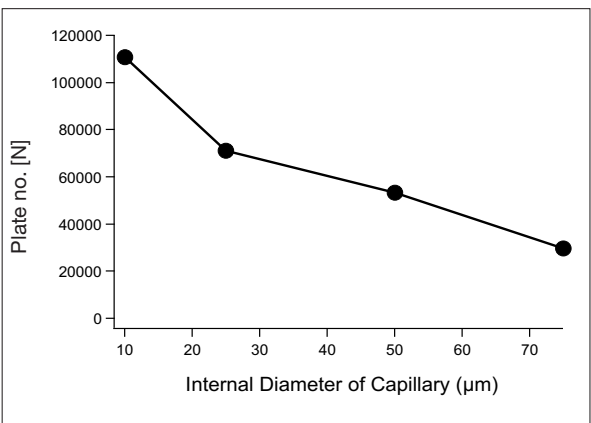

Fig. 3. Theoretical Plate Numbers $(\mathrm{N})$ according to the van Deemter theory as a measure of peak sharpness and separation efficiency vs. the internal diameter of the capillary for the injection of $\mathrm{Na}^{+}$at $100 \mu \mathrm{M}$.

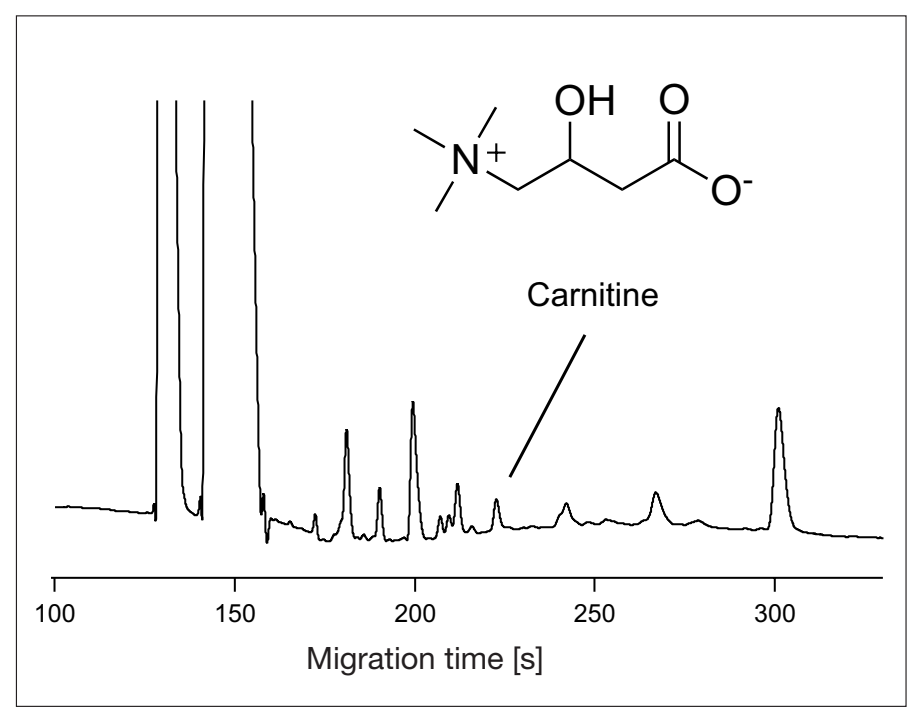

Fig. 4. Determination of carnitine in a sample of yoghurt by capillary zone electrophoresis with $\mathrm{C}^{4}$ D. ${ }^{[14]}$

obtained when going down to channels as narrow as $10 \mu \mathrm{m}$. This can be achieved with $C^{4} \mathrm{D}$ without significant deterioration of the sensitivity, as the detection limit was affected by less than a factor of $2(1.8 \mu \mathrm{M}$ vs. $3.0 \mu \mathrm{M}$ for the capillaries of 75 and 10 $\mu \mathrm{m}$ ID respectively).

As a typical application of $C E-C^{4} D$, the determination of carnitine, an essential nutrient, is illustrated in Fig. 4. Carnitine is generally difficult to quantify by analytical methods due to the fact that it is only very poorly UV-absorbing, it is a small molecule and has a zwitterionic nature. Often enzymatic methods coupled to colour forming auxiliary reactions are needed; alternatively if HPLC is to be used this is only possible after derivatization. $\mathrm{C}^{4} \mathrm{D}$ allows direct quantification in capillary electrophoresis following separation as a cation at a low $\mathrm{pH}$-value where the acid group of the molecule is protonated. ${ }^{[14]}$

The contactless detection method is also well suited for detection on microfabricated miniature separation platforms, and the simplicity of electrochemical detection methods is well matched to the downscaled devices. The use of $\mathrm{C}^{4} \mathrm{D}$ allows the employment of external electrodes, which do not have to be embedded or even attached to the separation chips as with the other electrochemical techniques, and this represents a significant simplification. ${ }^{[15,16]}$ In fact, except for the miniaturized manifold for separation, the entire system is electronic as further mechanical, optical or optoelectronic components are not required. The interaction with the sample is with the simple tool of two pairs of electrodes, one for separation, the other, which is not even in contact, for the detection.

This allows for unprecedented simplicity as modern electronics are compact, robust, inexpensive and consume little power. A pair of electrodes for detection on a separation channel of a microchip de-

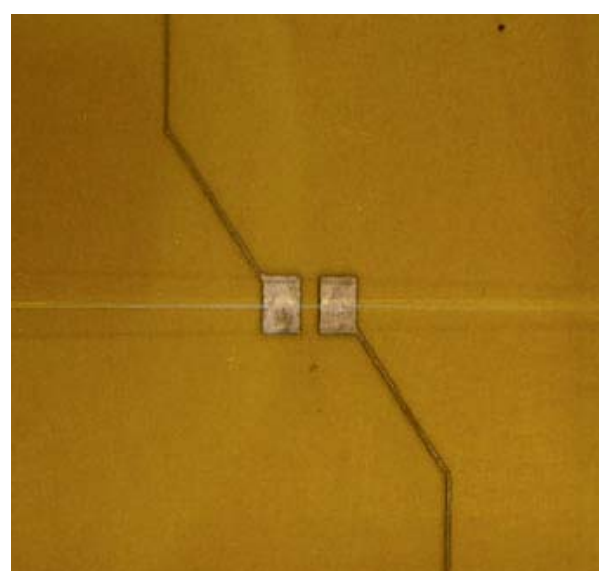

Fig. 5. Photo of planar electrodes located underneath the separation channel of an electrophoretic lab-on-chip device. The electrodes are about $1 \mathrm{~mm}$ squared, and the channel has a width of $50 \mu \mathrm{m}$.

vice is shown in the photograph in Fig. 5 . The analytical determination of the major inorganic ions in blood serum samples is a task carried out many millions of times daily as these are the standard blood electrolytes determined in clinical analysis for diagnostic purposes. The rapid quantification of these species is possible using microchip-CE- $\mathrm{C}^{4} \mathrm{D}^{[17]}$ and is illustrated as an example of the use of $C^{4} \mathrm{D}$ on lab-on-chip devices in Fig. 6.

\section{$C^{4}$ D for Capillary HPLC}

Despite the fact that HPLC is securely entrenched as the workhorse of industry for the analytical quantification of organic species, new detectors are still being sought. This is due to the fact that several important classes of analytes absorb only poorly in the UV-range and are thus not easily accessible. Due to the rising popularity of conductivity detection in $\mathrm{CE}, \mathrm{C}^{4} \mathrm{D}$ 


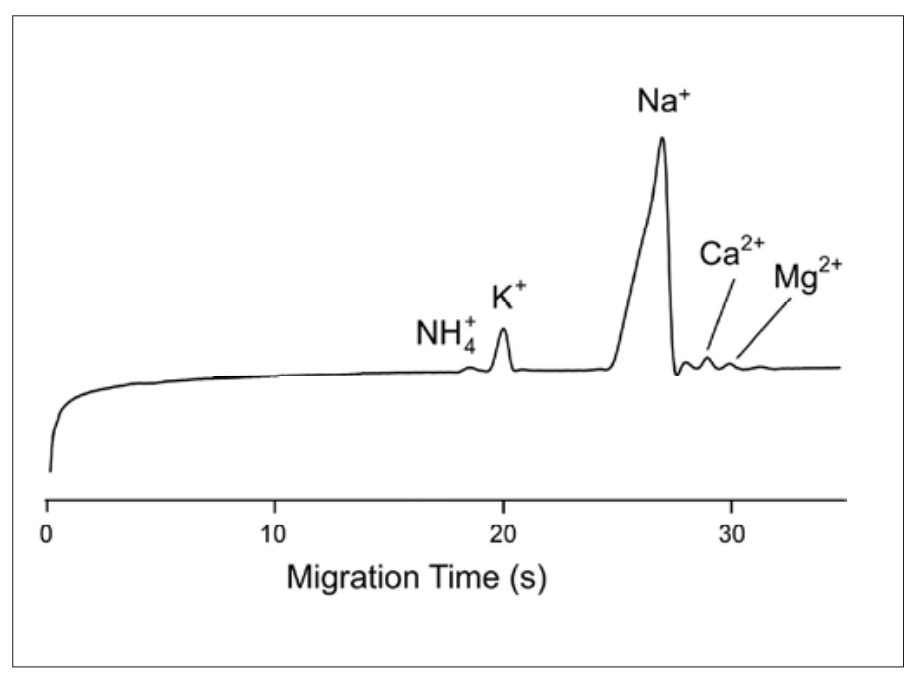

Fig. 6. Rapid separation of the major inorganic cations in a blood serum sample on a lab-on-chip device using $C^{4} D .{ }^{[17]}$

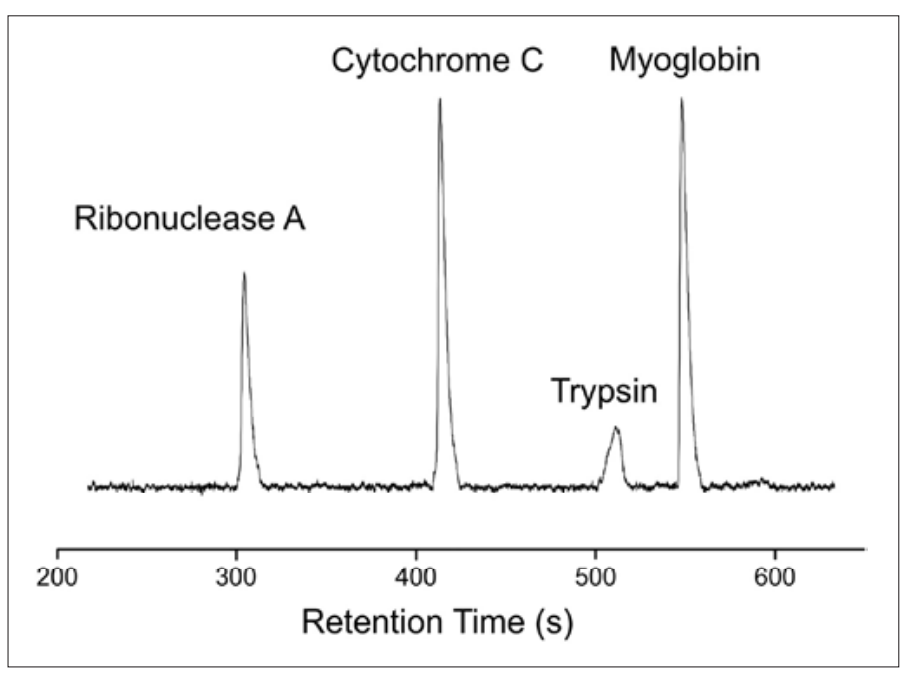

Fig. 7. Separation of a model mixture of proteins $(10 \mu \mathrm{g} / \mathrm{mL}$, except trypsin at $20 \mu \mathrm{g} / \mathrm{mL}$ ) in chromatography using a monolithic capillary column of $200 \mu \mathrm{m} \mathrm{ID}$ and $C^{4} D^{[20]}$ is increasingly also being investigated for use in chromatography and early studies have been reported by our group. ${ }^{[18,19]}$ The combination is of particular interest in the context of the current trend to capillary columns. The separation and detection of several proteins using a capillary column coupled to a $\mathrm{C}^{4} \mathrm{D}$-cell is shown in Fig. 7 as an illustration of the latter approach. ${ }^{[20]}$

\section{Acknowledgements}

The author would like to thank Pavel Kubán, Worapan Promsila and Than Duc Mai for providing the data presented.

Received: October 11, 2010

[1] R. Guchardi, P. C. Hauser, J. Anal. At. Spectrom 2003, 18, 1056.

[2] R. Guchardi, P. C. Hauser, J. Chromatogr. A 2004, 1033, 333.

[3] R. Guchardi, P. C. Hauser, The Analyst 2004, 129, 347.

[4] R. Guchardi, P. C. Hauser, J. Anal. At. Spectrom. 2004, 19, 945 .

[5] T. Kappes, P. C. Hauser, The Analyst 1999, 124, 1035.

[6] M. A. Schwarz, P. C. Hauser, J. Chromatogr. A
2001, 928, 225

[7] M. A. Schwarz, B. Galliker, K. Fluri, T. Kappes, P. C. Hauser, The Analyst 2001, 126, 147.

[8] L. Krčmová, A. Stjernlof, S. Mehlen, P. C. Hauser, S. Abele, B. Paull, M. Macka, The Analyst 2009, 134, 2394.

[9] S. Schmid, M. Macka, P. C. Hauser, The Analyst 2008, 133, 465 .

[10] P. Kubáň, P. C. Hauser, Electrophoresis 2004, $25,3387$.

[11] P. Kubáň, P. C. Hauser, Electrophoresis 2004, 25, 3398

[12] P. Kubáň, P. C. Hauser, Lab Chip 2005, 5, 407.

[13] P. Kubáň, P. C. Hauser, Electrophoresis 2009, 30, 176.

[14] W. Pormsila, S. Krähenbühl, P. C. Hauser, Electrophoresis 2010, 31, 2186.

[15] J. Tanyanyiwa, E. M. Abad-Villar, M. T. Fernández-Abedul, A. Costa-García, W. Hoffmann, A. E. Guber, D. Herrmann, A. Gerlach, N. Gottschlich, P. C. Hauser, The Analyst 2003, $128,1019$.

[16] J. Tanyanyiwa, P. C. Hauser, Anal. Chem. 2002, $74,6378$.

[17] Kubáň, P. C. Hauser, Lab Chip 2008, 8, 1829.

[18] Kubáň, P. C. Hauser, J. Chromatogr. A 2006, $1128,97$.

[19] P. Kubáň, E. M. Abad-Villar, P. C. Hauser, J. Chromatogr. A 2006, 1107, 159.

[20] P. Kubáň, P. C. Hauser, J. Chromatogr. A 2007, 1176, 185. 